\title{
Surgery for acute aortic dissection using the Chinese CRONUS stented elephant trunk technique: Experience with 252 patients
}

\author{
Hong-bing Wu, MD, Hao Zhang, MD, Zhi-wei Wang, PhD, Rui Hu, MD, Luo-cheng Li, MD, \\ Min Zhang, MD, and Xiao-ping Hu, MD
}

\begin{abstract}
Objectives: The elephant trunk method was introduced to treat aortic disease. There are a variety of modified elephant trunk methods, including the stented elephant trunk. We retrospectively reviewed our experience and evaluated the effectiveness of surgical treatment for acute aortic dissection using the Chinese CRONUS stented elephant trunk technique.
\end{abstract}

\begin{abstract}
Methods: From August 2005 to December 2012, 252 patients with acute aortic dissection underwent surgical treatment using the Chinese CRONUS stented elephant trunk technique at the Renmin Hospital of Wuhan University. We review the characteristics of the patients, the surgical method, and the prognosis. Furthermore, we modified the stented elephant trunk technique to simplify the surgical procedure using stented elephant trunk fenestration in 81 patients.
\end{abstract}

Results: The procedure was technically successful in all patients. The mean duration of cardiopulmonary bypass, myocardial ischemia, and circulatory arrest was $158 \pm 34$ minutes, $98 \pm 24$ minutes, and $27 \pm 9$ minutes, respectively. The mean stay in the intensive care unit was $74 \pm 11$ hours. The in-hospital mortality rate was $3.2 \%(8 / 252)$. A $92.2 \%$ (225/244) follow-up rate was achieved. Five patients died during follow-up. The diameter of the descending aorta significantly decreased in 173 patients $(78.6 \%)$, did not change 39 patients $(17.7 \%)$, and dilated in 8 patients $(3.7 \%)$.

Conclusions: In surgery for acute aortic dissection, the Chinese CRONUS stented elephant trunk technique had a low prevalence of morbidity and mortality in our patients. The satisfactory effects demonstrated that the technique is safe and effective in closing the residual false lumen of the descending aorta. Stented elephant trunk fenestration could further simplify the surgical procedure with minimal invasion. (J Thorac Cardiovasc Surg 2014;148:2132-8)

See related commentary on pages 2138-9.

Although the outcomes of surgical treatment for acute type A aortic dissection (ATAAD) have greatly improved in recent years, it is still associated with high mortality and morbidity. ${ }^{1}$ Appropriate surgical management of ATAAD is a critical factor for achieving a satisfactory outcome, but the choice of optimal procedure is controversial. Total arch replacement (TAR) combined with stented elephant trunk (SET) implantation demonstrated the superiority

\footnotetext{
From the Department of Cardiovascular Surgery, Renmin Hospital of Wuhan University, Wuhan Hubei, People's Republic of China.

This work was supported by grants from the Natural Science Foundation of Hubei Province (China) (No. 2008CHB421).

Disclosures: Authors have nothing to disclose with regard to commercial support.

Hong-bing Wu and Hao Zhang are co-primary authors.

Received for publication Aug 28, 2013; revisions received Nov 10, 2013; accepted for publication Dec 5, 2013; available ahead of print Feb 7, 2014

Address for reprints: Zhi-wei Wang, MD, Department of Cardiovascular Surgery, Renmin Hospital of Wuhan University, Jiefang Rd 238, Wuhan 430060, People's Republic of China (E-mail: hbwu2008@163.com).

0022-5223/\$36.00

Copyright (c) 2014 by The American Association for Thoracic Surgery

http://dx.doi.org/10.1016/j.jtcvs.2013.12.007
}

of the combination of the surgical and interventional approaches while avoiding the weaknesses associated with the individual methods. The encouraging surgical results could enable this procedure to become the new "standard" therapy for type A dissection involving repair of the aortic arch. ${ }^{2}$ The SET technique has an important role to play in this process. According to a patient's condition, surgical treatment, conservative therapy, and endovascular exclusion can be selected for acute type B aortic dissection (ATBAD). The optimal surgical approach for ATBAD is still being debated. The SET technique has been applied to treat complicated type B aortic dissection, ${ }^{3}$ and numerous satisfactory results have been reported by investigators. ${ }^{3}$ The SET technique has been proposed as an efficient means to treat complicated type B aortic dissection. Therefore, the SET technique dominates the treatment of aortic dissection. ${ }^{4}$ Because SET has been used in the treatment of aortic disease only in the last 15 years and was first used in the treatment of aneurysm, ${ }^{5}$ clinical experience is severely limited. We retrospectively reviewed our experience and evaluated the effectiveness of surgical treatment for acute aortic dissection using the Chinese CRONUS SET technique. In addition, we performed this surgical procedure with some technical modifications in suitable cases. 

Abbreviations and Acronyms
ATAAD $=$ acute type $\mathrm{A}$ aortic dissection
ATBAD $=$ acute type $\mathrm{B}$ aortic dissection
$\mathrm{CABG}=$ coronary artery bypass grafting
$\mathrm{CPB}=$ cardiopulmonary bypass
CTA = computed tomography angiography
FET = frozen elephant trunk
SET = stented elephant trunk
TAR $=$ total arch replacement
TEVAR $=$ thoracic endovascular aortic repair

\section{MATERIALS AND METHODS}

The study protocol was approved by the Committee for the Protection of Human Subjects at the Renmin Hospital of Wuhan University, with patient consent waived.

\section{Patient Population}

From August 2005 to December 2012, 252 patients with acute aortic dissection (type $A=161$, type $B=91$ ), underwent surgical treatment using the Chinese CRONUS SET technique at the Department of Cardiovascular Surgery, Renmin Hospital of Wuhan University (Wuhan, China). Inclusion criteria for ATAAD were as follows: (1) acute onset (from onset to hospitalization $<2$ weeks ); (2) use of femoral artery perfusion (patients with subclavian artery perfusion or ascending aorta perfusion were excluded); (3) use of bilateral antegrade cerebral perfusion (patients with unilateral antegrade or retrograde cerebral perfusion were excluded); and (4) no surgery contraindications. Inclusion criteria for ATBAD were as follows: (1) acute onset (from onset to hospitalization $<2$ weeks); (2) patients had a tortuous descending aorta or true lumen less than false lumen, and thoracic endovascular aortic repair (TEVAR) was abandoned because of increased risk; (3) a second surgery may be required (descending aortic dilatation); and (4) no surgery contraindications. The mean age of these patients was $55.2 \pm 2.5$ years (range, 27-75 years), and 212 patients were male. Retrosternal chest pain and back pain were the common presenting symptoms. Twenty patients had Marfan syndrome. Two patients experienced retrograde type A aortic dissection after endovascular stent graft placement for type B dissection. Ascending aortic dissection evolved into a rupture in 1 patient. The diagnosis was based on 64-slice computed tomography angiography (CTA) and echocardiography. TAR combined with the Chinese CRONUS SET technique was carried out in patients with type A dissection. The Chinese CRONUS SET technique was conducted in patients with type B. To minimize bleeding from anastomosis, some patients received SET fenestration according to the intraoperative exploration. SET fenestration also can elevate the anastomosis plane and avoid the difficulty of anastomosis. Indications of SET fenestration are as follows: (1) The distance between the intimal tear and the orifice of left subclavian artery is greater than $1.5 \mathrm{~cm}$; (2) there is no dissection in the range of ostia of left subclavian artery, and the diameter is greater than $1.0 \mathrm{~cm}$; (3) no stenosis in the ostia of left subclavian artery; and (4) the diameter of descending aorta is less than $3.0 \mathrm{~cm}$. Patients' preoperative characteristics are listed in Table 1. Postoperative CTA was performed routinely to assess the state of the residual false lumen during follow-up.

\section{Chinese CRONUS Stented Graft}

The Chinese CRONUS stented graft device was made by MicroPort Medical (Shanghai) Co, Ltd, Shanghai, China. This stent graft consists of a polyester vascular graft and a metal stent. The polyester vascular graft is longer than the stent and used to cover the stent. The stent graft is made in a bound and compressed state. The delivery system consists of a catheter sheath and a pulling ring. The diameter varies from 26 to $32 \mathrm{~mm}$, and the length ranges from 80 to $100 \mathrm{~mm}$. Both ends were presented as a nonstent sewing edge. The pulling ring could steadily release the stented portion while holding the handle.

\section{Surgical Procedure}

All procedures were performed in patients under general anesthesia and via a standard median sternotomy. The arterial blood pressure of both the upper and lower limbs was monitored. In patients with ATAAD, the procedures were carried out by total cardiopulmonary bypass (CPB) with bilateral antegrade cerebral perfusion. Cannulation of the femoral artery was used for CPB. The right atrium was cannulated with a dual-stage atriocaval cannula. The temperature decreased uniformly after $\mathrm{CPB}$ was begun. When the nasopharyngeal temperature declined to $28^{\circ} \mathrm{C}$ to $30^{\circ} \mathrm{C}$, the ascending aorta was crossclamped and the heart was arrested with the perfusion of a cold blood cardioplegic solution. During cooling, the ascending aorta just distal to the sinotubular junction was transected and aortic root procedures were performed, including Bentall, Wheat, David, and coronary artery bypass grafting ( $\mathrm{CABG}$ ) according to the surgical inspection of the involvement of the aortic root, including the aortic valve leaflets and coronary ostia. When the nasopharyngeal temperature reached $18^{\circ} \mathrm{C}$ to $20^{\circ} \mathrm{C}$, circulatory arrest was initiated. The aortic arch was opened longitudinally. Bilateral antegrade cerebral perfusion was performed through the brachiocephalic trunk and left common carotid artery, and the brain was perfused. The flow rate was maintained at 5 to $10 \mathrm{~mL} / \mathrm{kg} / \mathrm{min}$, and perfusion pressure was maintained at 30 to $40 \mathrm{~mm} \mathrm{Hg}$. Ice packs were placed around the head. Transection of the proximal descending thoracic aorta was made below the origin of left subclavian artery. Recurrent laryngeal and phrenic nerves were protected carefully. The Chinese CRONUS stented graft was placed into the appropriate segment of the descending thoracic aorta. The expansion of the stented graft was triggered by pulling the release element through the pulling ring. The proximal end of the stented graft and aortic wall was anastomosed together to a 4-branched prosthetic graft, using continuous suturing with 4-0 polypropylene sutures. When this anastomosis was completed, antegrade systemic perfusion was started through the perfusion limb of the 4-branched prosthetic graft that connected to the CPB circuit. The reconstruction of arch vessels was performed as follows: The left subclavian artery, left common carotid artery, and brachiocephalic trunk were anastomosed to the side branches of the branched graft. The clamp was removed to allow perfusion once the anastomosis was completed. As soon as the left common carotid artery anastomosis was completed, rewarming was started. When the reconstruction of all arch vessels was finished, the proximal anastomosis of the ascending aorta was performed. There were 15 patients whose aortic arches were replaced using a separate Dacron tube graft first, and then the supra-aortic branches (brachiocephalic trunk, left common carotid, and left subclavian arteries) were anastomosed to Dacron tube graft as a single tissue patch.

In patients with ATBAD, the procedures were carried out by total CPB without cerebral perfusion. When the nasopharyngeal temperature reached $18^{\circ} \mathrm{C}$ to $20^{\circ} \mathrm{C}$, circulatory arrest was initiated. An incision (15-20 mm long) was made between the left common carotid and left subclavian arteries. The stented graft was placed into the appropriate segment of descending thoracic aorta to close the tear site and attached to the wall of descending thoracic aorta using continuous suturing with 4-0 polypropylene sutures.

\section{Modified Stented Elephant Trunk Technique}

We modified the described SET technique to simplify the surgical procedure using SET fenestration in 81 patients, 51 with type A aortic dissection and 30 with type B aortic dissection. SET fenestration was performed in those with type A aortic dissection as follows (Figure 1): Under circulatory arrest at a nasopharyngeal temperature of $18^{\circ} \mathrm{C}$ to $20^{\circ} \mathrm{C}$, the aortic arch was transected between the left common carotid and left subclavian arteries. The stented graft was placed into the descending thoracic aorta. The nonstent 
TABLE 1. Patient characteristics

\begin{tabular}{lcc}
\hline \multicolumn{1}{c}{ Variables } & No. & Percent \\
\hline Patients & 252 & 100 \\
Type A & 161 & 63.9 \\
Type B & 91 & 36.1 \\
Age (mean \pm SD) y & $55.2 \pm 2.5$ & \\
Male & 211 & 83.7 \\
Female & 41 & 16.3 \\
Hypertension & 136 & 54.0 \\
Cardiac valve disease & 124 & 49.2 \\
Pleural effusion & 114 & 45.2 \\
Microscopic hematuria & 51 & 20.2 \\
Hemopericardium & 49 & 19.4 \\
DM & 26 & 10.3 \\
Renal dysfunction & 16 & 6.3 \\
MFS & 20 & 7.9 \\
COPD & 7 & 2.8 \\
CHD & 7 & 2.8 \\
Previous cerebrovascular accident & 3 & 1.2 \\
Asthma & 3 & 1.2 \\
Previous ESGP & 2 & 0.8 \\
Previous Bentall surgery & 2 & 0.8 \\
Limb ischemia & 2 & 0.8 \\
Rupture & 1 & 0.4 \\
Paraplegia & 1 & 0.4 \\
\hline CHD, Cora & & \\
\hline
\end{tabular}

$C H D$, Coronary heart disease; $C O P D$, chronic obstructive pulmonary disease; $D M$, diabetes mellitus; ESGP, endovascular stent graft placement; $M F S$, Marfan syndrome; $S D$, standard deviation.

sewing edge overstepped the orifice of the left subclavian artery by more than $10 \mathrm{~mm}$. The part of the stent that covered the polyester vascular graft closed the left subclavian artery. A fenestration, 5 to $8 \mathrm{~mm}$ in diameter, was taken in the stented graft contrary to the left subclavian artery by a cauter. The distal end of the 4-branched prosthetic graft was anastomosed to the transected transverse aortic arch containing the inserted stented graft. After distal anastomosis was completed, antegrade systemic perfusion was begun through one of the graft branches. The brachiocephalic trunk and left common carotid were respectively anastomosed to a 4-branched prosthetic graft. The reconstruction of all arch vessels was finished.

SET fenestration was performed in type B aortic dissection as follows: Under circulatory arrest at a nasopharyngeal temperature of $18^{\circ} \mathrm{C}$ to $20^{\circ} \mathrm{C}$, an incision (15-20 mm long) was made between the left common carotid and left subclavian arteries. The stented graft was placed into the appropriate segment of the descending thoracic aorta to close the tear site and left subclavian artery. A fenestration, 5 to $8 \mathrm{~mm}$ in diameter, contrary to the left subclavian artery, was made in the stented graft by a cauter. The stented graft was attached to the wall of descending thoracic as described earlier.

\section{Data Collection and Follow-up}

Hospital records for all patients were retrospectively reviewed. Continuous variables are presented as mean \pm standard deviation, and categoric variables are presented as numbers and percentages. All live patients were followed up by direct interviews in our outpatient department or telephone. They were followed up by CTA before discharge, at 6 months postoperatively, and annually thereafter.

\section{RESULTS}

\section{Primary Intimal Tear Site}

In 161 patients with type A aortic dissection, the primary intimal tear site was at the ascending aorta in 141, at the arch in 5 , and at the proximal descending aorta in 11 . An entry tear was not detected in 4 patients, and 18 patients had multiple intimal tears. The primary intimal tear was at the proximal descending aorta in 91 patients with type B aortic dissection.

\section{Surgical Technique}

The procedure was technically successful in all patients, including TAR, SET, fenestration, ascending aorta replacement, and aortic root operation (Bentall, Wheat, David, and CABG). The SET procedure was performed in 252 patients, Bentall in 70, ascending aorta replacement in 37 , Wheat in 33, David in 21, CABG in 7, and fenestration in 81 . The surgical procedures are presented in Table 2. The mean duration of $\mathrm{CPB}$ was $158 \pm 34$ minutes, the mean duration of myocardial ischemia was $98 \pm 24$ minutes, and the mean duration of circulatory arrest was $27 \pm 9$ minutes. The mean stay in the intensive care unit was $74 \pm 11$ hours. The perioperative data are listed in Table 3.

\section{Early Mortality and Hospital Morbidity}

The in-hospital mortality rate was $3.2 \%$ (8/252). Of these fatalities, the cause was multiple organ failure in 4 patients, infectious shock in 2 patients, hemorrhagic shock in 1 patient, and infectious endocarditis in 1 patient. Complications included transient neurologic deficit in 51 patients $(20.2 \%)$ and cerebrovascular accident in 4 patients $(1.6 \%$, cerebral embolism in 2 and cerebral hemorrhage in 2). Nine patients underwent tracheotomy. Reexploration of mediastinum was indicated in 4 patients for bleeding, and 2 patients temporarily experienced hoarseness. Three patients had renal failure that was cured by hemodialysis. One patient underwent reoperation for sternal dehiscence. One patient had unilateral limb ischemia and received femoro-femoral bypass grafting. No episode of paraplegia or paralysis was recorded in all groups, and all other patients recovered uneventfully. The overall hospital mortality and morbidity are described in Table 4.

\section{Follow-up}

Clinical data were obtained by personal and telephone interviews. All complications were recorded. A 92.2\% (225/244) follow-up rate was achieved. The mean followup was $30 \pm 10$ months (range, 6-96 months). Five patients died during follow-up. One patient died of cerebral hemorrhage after 4 months because of inappropriate anticoagulation during follow-up, 1 patient died of multiorgan failure caused by severe septicopyemia, 1 patient died of acute myocardial infarction, 1 patient with cerebrovascular accident died of multiorgan failure 18 days after discharge, and 1 patient died of chronic renal failure. These patients, with cerebrovascular accident in 3 and hoarseness in 2, had improved.

CTA was performed in 220 of 244 hospital survivors who had been followed for more than 1 year. At 1 year 

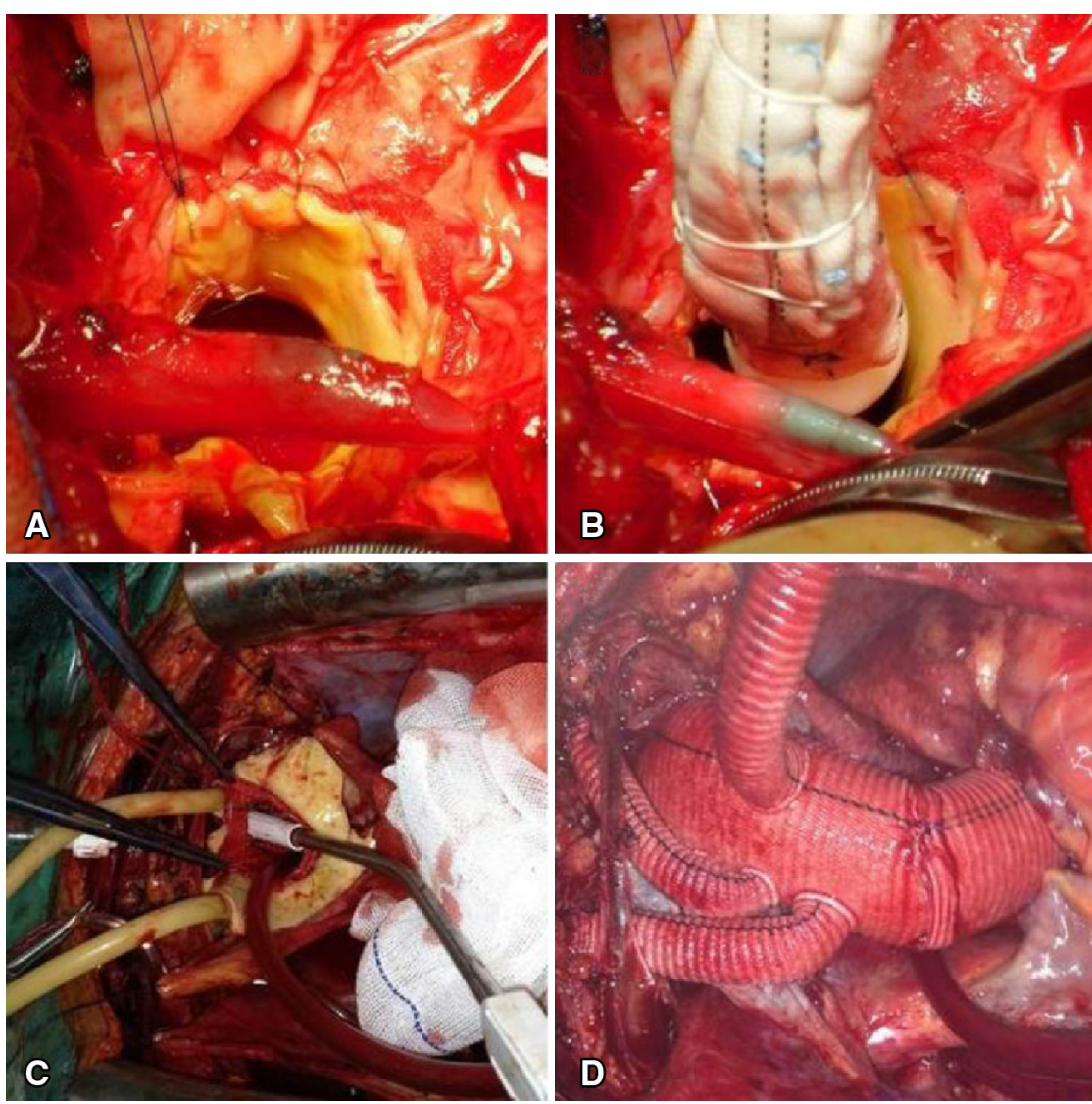

FIGURE 1. SET fenestration in a patient with ATAAD. A, Transection of the aortic arch was performed between the left common carotid and left subclavian arteries. B, The stented graft was placed into the descending thoracic aorta. C, Completion of SET fenestration (suction tube pointing). $\mathrm{D}$, Completion of reconstruction of all arch vessels.

postoperatively, 17 patients exhibited a completely false lumen obliteration and approximate normal aorta at 1 year postoperatively. The diameter of the residual false lumen in the descending aorta significantly decreased in 173 patients, did not change in 39 patients, and dilated in 8 patients. No endoleaks were found in patients who underwent SET fenestration. The left subclavian arteries had good blood circulation (Figure 2). Among patients with

TABLE 2. Surgical procedure

\begin{tabular}{lr}
\hline Surgical procedure & No. \\
\hline Type A dissection & 161 \\
AAR + SET & 37 \\
Bentall + TAR + SET & 70 \\
Wheat + TAR + SET & 33 \\
David + TAR + SET & 21 \\
Fenestration & 51 \\
CABG & 5 \\
Type B dissection & 91 \\
SET & 91 \\
Fenestration & 30 \\
CABG & 2 \\
\hline
\end{tabular}

$A A R$, Ascending aorta replacement; $C A B G$, coronary artery bypass grafting; $S E T$, stented elephant trunk; TAR, total arch replacement. dilation, 1 with Marfan syndrome showed progressive dilation of the residual false lumen during follow-up. This patient recovered after the replacement of the remaining descending and abdominal aortas. The status of the descending aortas is shown in Table 5.

\section{DISCUSSION}

ATAAD is a surgical emergency and still remains a research hotspot. ${ }^{6}$ With the development of surgical techniques, the outcomes of surgical treatment for ATAAD have greatly improved over the years, but it still carries

TABLE 3. Perioperative data

\begin{tabular}{lc}
\hline \multicolumn{1}{c}{ Variables } & Mean \pm SD \\
\hline Total CPB (min) & $158 \pm 34$ \\
Myocardial ischemia (min) & $98 \pm 24$ \\
Circulatory arrest (min) & $27 \pm 9$ \\
Nasopharyngeal temperature $\left({ }^{\circ} \mathrm{C}\right)$ & $19 \pm 3$ \\
Ventilation time (h) & $20 \pm 6$ \\
ICU time (h) & $74 \pm 11$ \\
In-hospital time (d) & $27 \pm 11$ \\
Drainage in first day (mL) & $435 \pm 109$ \\
\hline$C P B$, Cardiopulmonary bypass; $I C U$, intensive care unit; $S D$, standard deviation.
\end{tabular}


TABLE 4. Hospital mortality and morbidity

\begin{tabular}{lc}
\multicolumn{1}{c}{ Variables } & Valve \\
\hline In-hospital mortality & $8(3.2 \%)$ \\
TND & $51(20.2 \%)$ \\
Tracheotomy & $9(3.6 \%)$ \\
CVA & $4(1.6 \%)$ \\
Redo for bleeding & $4(1.6 \%)$ \\
Renal dysfunction & $3(1.2 \%)$ \\
Hoarseness & $2(0.8 \%)$ \\
Limb ischemia & $1(0.4 \%)$ \\
Sternal dehiscence & $1(0.4 \%)$ \\
\hline$C V A$, Cerbosch
\end{tabular}

$C V A$, Cerebrovascular accident; $T N D$, transient neurologic deficit.

high mortality and morbidity. ${ }^{7}$ The elephant trunk plays a critical role in the treatment of type A aortic dissection. The conventional elephant trunk ${ }^{8}$ was introduced to facilitate subsequent surgery on the distal aorta in type A dissection. This technique reduced operative risks but was associated with cumulative mortality rates of $6.9 \%$ for the first stage and $7.5 \%$ for the second stage. ${ }^{9}$ Placing the prosthetic graft into the true lumen of a type A dissection was difficult. ${ }^{10}$ The prosthetic graft also had a "flapping" action. Deaths caused by rupture of the remaining aortic aneurysm in the interval between the 2 procedures have occurred, and some patients did not return for the second stage of the procedure. ${ }^{11}$
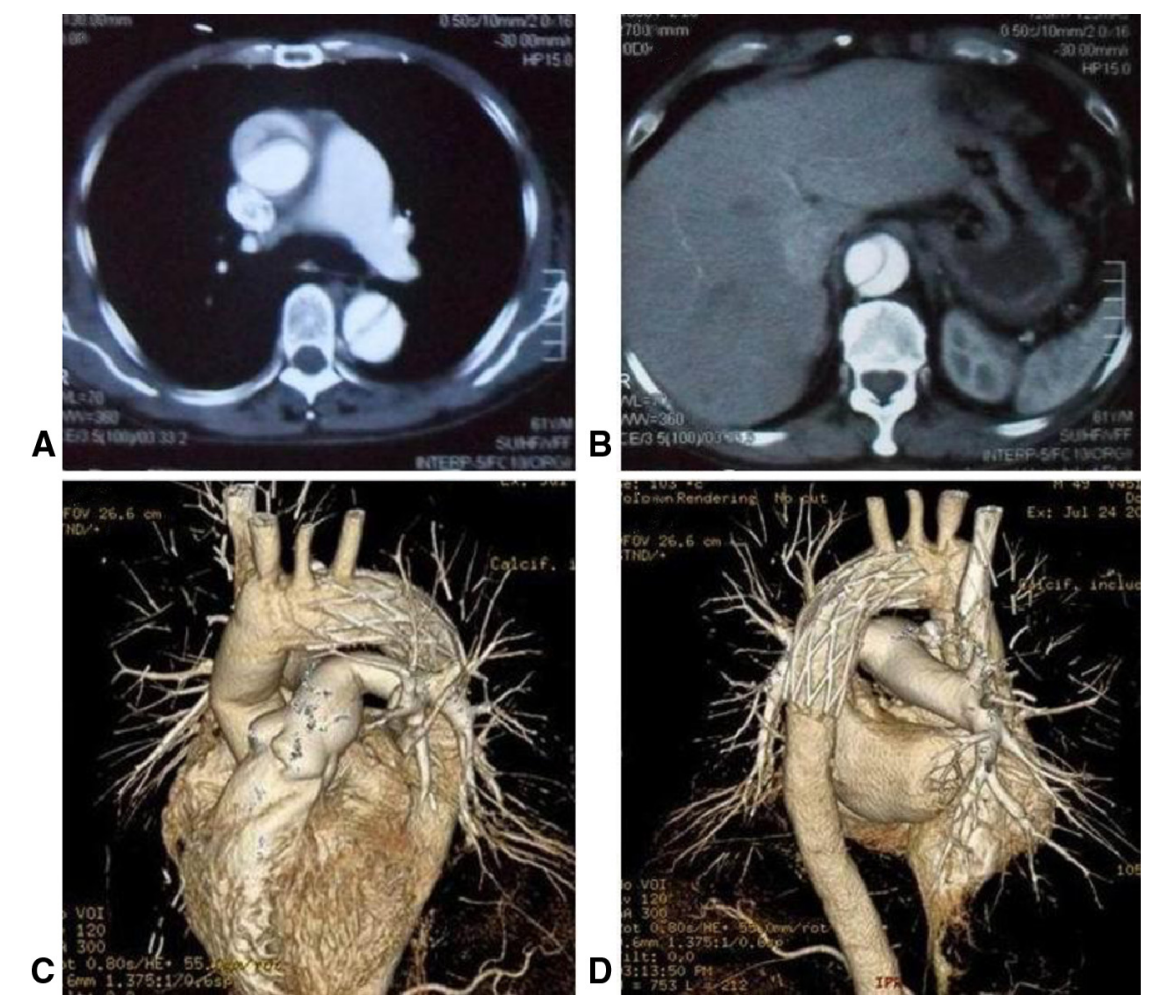
colleagues ${ }^{17}$ to treat type A dissection.

P

To avoid the complications discussed, Kato and colleagues $^{12}$ and Karck and colleagues ${ }^{13}$ designed a selfexpandable stent to sustain the distal end of the elephant trunk (frozen elephant trunk [FET]). The proximal end of the FET was nonstented and consisted of a Dacron sleeve ready for conventional surgical handling. The FET is helpful to achieve complete thrombosis of the false lumen in the descending thoracic aorta ${ }^{14}$ and may reduce the necessity for further operations to manage a residual lumen. ${ }^{15}$ However, only the distal part of the prosthetic graft segment can be sustained by a self-expandable stent. It was difficult to select an appropriate size of the FET, which introduced a high risk of damage to the intima. ${ }^{15}$ Endovascular placement of a covered stent graft and surgical procedure were combined to treat type A dissection. The covered stent was similar to the self-expandable stent, but the covered stent was delivered in a retrograde fashion via the femoral artery and thus made the relevant complication inevitable. ${ }^{16}$ The corresponding complications cannot be prevented. Furthermore, there is no extravascular graft at the end of the stent graft to be used for sewing. Therefore, the SET procedure, with the advantages of good intraoperative handing and postoperative recovery, was introduced by Liu and

We performed surgical treatment for aortic dissection with the Chinese CRONUS SET technique. This procedure

FIGURE 2. CTA of an ATAAD. A and B, Preoperative CTA shows ATAAD involving the ascending, descending, and abdominal aortas. C and D, CTA after SET fenestration 1 year postoperatively shows the stented graft fully expanded and the aortic wall remodeled to a normal shape. No endoleaks were found. Left subclavian artery (fenestration) shows good blood circulation. 
TABLE 5. Status of the descending aorta

\begin{tabular}{lccr}
\hline \multicolumn{1}{c}{ Variables $(\mathbf{m m})$} & Preoperative & Postoperative & n \\
\hline Diameter of DTA & $35.06 \pm 4.19$ & $30.11 \pm 2.92$ & 220 \\
Diameter of AA & $29.02 \pm 3.13$ & $25.05 \pm 2.28$ & 220 \\
Decrease of DTA & $35.04 \pm 4.16$ & $28.56 \pm 3.24$ & 173 \\
No change in DTA & $35.00 \pm 3.41$ & $35.07 \pm 4.10$ & 39 \\
Enlargement of DTA & $35.79 \pm 3.81$ & $38.03 \pm 4.34$ & 8 \\
\hline
\end{tabular}

$A A$, Abdominal aorta; $D T A$, descending thoracic aorta.

has the following advantages: First, precise implantation of the SET into the true lumen of the distal aorta is easy with the antegrade approach under direct vision. The intimal tear can be accurately closed by the stent. Second, the released stent graft can gradually enlarge the true lumen versus shrink the false lumen. The distal artery can quickly restore normal blood flow. Third, we use continuous suture to ensure a firm fixation of the 4-branch prosthetic graft to the distal end. Some complications of the covered stent, such as endoleaks and stent translocation, were avoided. Fourth, injury to the recurrent laryngeal nerve could be prevented in addition to the decreased difficulty of distal anastomosis in the descending aorta; thus, it simplified conventional TAR. Fifth, an extravascular graft at the end of the stent graft was suitable for suturing if a secondary operation was needed.

We modified the SET technique to further simplify the surgical procedure using SET fenestration in 81 patients. The purpose of SET fenestration is to simplify the surgical procedure: (1) Anastomotic stoma and bleeding are reduced because of a more superficial site for anastomosis. (2) The less operative procedure of aortic arch results in better protection of the recurrent laryngeal and phrenic nerves. (3) Further decrease of operation time and duration of $\mathrm{CPB}$ and circulatory arrest results in fewer operative injuries. The indication has been described in the patient population. If it is less $1.5 \mathrm{~cm}$ or the left subclavian artery is involved, the procedure cannot be performed. Otherwise, endoleaks are possible in this condition.

ATBAD is a life-threatening disease. The surgical treatment using a prosthetic graft has been a conventional treatment for ATBAD. During the past decade, TEVAR, a less invasive and potentially safer technique, has been increasingly used to treat this condition. TEVAR can significantly reduce the short-term mortality of ATBAD. ${ }^{18}$ However, TEVAR has not displayed superiority regarding postoperative complications and long-term mortality. A number of complications have been reported. ${ }^{19}$ TEVAR can be weakly recommended as an alternative for the selective treatment of ATBAD but cannot always be used in case of surgery. ${ }^{20}$ The appropriate approach for patients with type $\mathrm{B}$ dissection also remains controversial, and the surgical treatment remains a challenge. The SET technique has been used in the treatment of type B aortic dissection. We performed the SET technique to treat type B dissection using the Chinese CRONUS stented graft. The technique has the following advantages: (1) The stent is easily implanted into the descending aorta in just a few minutes, so it can be successfully completed without cerebral perfusion. (2) The incision on the aorta is small with minimal bleeding. (3) It is convenient to manage if aortic dissection progresses to type A during the operation. (4) When the left subclavian artery is located deep in the chest, the operation might be problematic. SET fenestration can be undertaken to simplify the operation. The indication is type B aortic dissection with multiple intimal tears, limited space of true lumen, severe tortuous aorta, and spiral dissection. Choosing an appropriate model stented graft for avoiding endoleaks, ischemia injury to organs, and paraplegia is critical. The profile of the stent graft is determined with the diameter of the artery and the length of the lesion. The diameter should exceed the diameter of the landing zone by at least $10 \%$ to $15 \%$. The length also is determined with the diameter. A short stent graft is more suitable for patients with a small abdominal aortic diameter, whereas a longer stent graft would accommodate a large baseline abdominal aortic diameter. ${ }^{21}$

\section{CONCLUSIONS}

In surgery for aortic dissection, the Chinese CRONUS SET technique demonstrated a low prevalence of morbidity and mortality in our patients. The satisfactory effects demonstrated that the technique is safe and effective in closing the residual false lumen of the descending aorta. SET fenestration could further simplify the surgical procedure with minimal invasion.

\section{References}

1. Trimarchi S, Nienaber CA, Rampoldi V, Myrmel T, Suzuki T, Mehta RH, et al. Contemporary results of surgery in acute type A aortic dissection: The International Registry of Acute Aortic Dissection experience. J Thorac Cardiovasc Surg. 2005; 129:112-22.

2. Sun L, Qi R, Zhu J, Liu Y, Zheng J. Total arch replacement combined with stented elephant trunk implantation: a new "standard" therapy for type a dissection involving repair of the aortic arch? Circulation. 2011;123:971-8.

3. Li B, Sun L, Chang Q, Zhu J, Yu C, Liu Y, et al. Total arch replacement with stented elephant trunk technique: a proposed treatment for complicated Stanford type B aortic dissection. Card Surg. 2009;24:704-9.

4. Svensson LG, Rushing GD, Valenzuela ES, Rafael AE, Batizy LH, Blackstone EH, et al. Modifications, classification, and outcomes of elephanttrunk procedures. Ann Thorac Surg. 2013;96:548-58.

5. Suto Y, Yasuda K, Shiiya N, Murashita T, Kawasaki M, Imamura M, et al. Stented elephant trunk procedure for an extensive aneurysm involving dista aortic arch and descending aorta. J Thorac Cardiovasc Surg. 1996;112:1389-90.

6. Qian H, Hu J, Du L, Xue Y, Meng W, Zhang EY. Modified hypothermic circulatory arrest for emergent repair of acute aortic dissection type A: a single-center experience. J Cardiothorac Surg. 2013;8:125-31.

7. Pagni S, Ganzel BL, Trivedi JR, Singh R, Mascio CE, Austin EH, et al. Early and midterm outcomes following surgery for acute type A aortic dissection. J Card Surg. 2013 [Epub ahead of print].

8. Borst HG, Walterbusch G, Schaps D. Extensive aortic replacement using "elephant trunk" prosthesis. Thorac Cardiovasc Surg. 1983;31:37-40.

9. Miyamoto Y. Elephant trunk technique for hybrid aortic arch repair. Gen Thorac Cardiovasc Surg. 2013 [Epub ahead of print].

10. Kieffer E, Koskas F, Godet G, Bertrand M, Bahnini A, Benhamou AC, et al. Treatment of aortic arch dissection using the elephant trunk technique. Ann Vasc Surg. 2000;14:612-9. 
11. Estrera AL, Miller CC, Porat EF, Huynh TT, Winnerkvist A, Safi HJ. Staged repair of extensive aortic aneurysms. Ann Thorac Surg. 2002;74:S1803-5.

12. Kato M, Ohnishi K, Kaneko M, Ueda T, Kishi D, Mizushima T, et al. New graftimplanting method for thoracic aortic aneurysm or dissection with a stented graft. Circulation. 1996;94(Suppl):II188-93.

13. Karck M, Chavan A, Hagl C, Friedrich H, Galanski M, Haverich A. The frozen elephant trunk technique: a new treatment for thoracic aortic aneurysms. J Thorac Cardiovasc Surg. 2003;125:1550-3.

14. Uchida N, Ishihara H, Shibamura H, Ozawa M. Midterm results of extensive primary repair of the thoracic aorta by means of total arch replacement with open stent graft placement for an acute type A aortic dissection. J Thorac Cardiovasc Surg. 2006;131:862-7.

15. Uchida N, Shibamura H, Katayama A, Shimada N, Sutoh M, Ishihara H. Operative strategy for acute type a aortic dissection: ascending aortic or hemiarch versus total arch replacement with frozen elephant trunk. Ann Thorac Surg. 2009;87:773-7.

16. Liu JC, Zhang JZ, Yang J, Zuo J, Zhang JB, Yu SQ, et al. Combined interventional and surgical treatment for acute aortic type a dissection. Int J Surg. 2008;6:151-6.
17. Liu ZG, Sun LZ, Chang Q, Zhu JM, Dong C, Yu CT, et al. Should the "elephant trunk" be skeletonized? Total arch replacement combined with stented elephant trunk implantation for Stanford type A aortic dissection. J Thorac Cardiovasc Surg. 2006;131:107-13.

18. Narayan P, Wong A, Davies I, Angelini GD, Bryan AJ, Wilde P, et al. Thoracic endovascular repair versus open surgical repair - which is the more cost-effective intervention for descending thoracic aortic pathologies? Eur J Cardiothorac Surg. 2011;40:869-74.

19. Nakao M, Yamashiro M, Matsumura Y, Yoshitake M, Tanaka K, Sakamoto Y, et al. Lower body ischemia due to bending of the stent after hybrid treatment for chronic Stanford type B aortic dissection. Kyobu Geka. 2013;66:791-4.

20. Hao Z, Zhi-Wei W, Zhen Z, Xiao-Ping H, Hong-Bing W, Yi G. Endovascular stent-graft placement or open surgery for the treatment of acute type B aortic dissection: a meta-analysis. Ann Vasc Surg. 2012;26:454-61.

21. Lee M, Lee DY, Kim MD, Lee MS, Won JY, Park SI, et al. Outcomes of endovascular management for complicated chronic type B aortic dissection: effect of the extent of stent graft coverage and anatomic properties of aortic dissection. $J$ Vasc Interv Radiol. 2013;24:1451-60.

\title{
EDITORIAL COMMENTARY
}

\section{Another approach to dissection involving the aortic arch}

\author{
Lars G. Svensson, MD, PhD
}

See related article on pages 2132-8.

The best approach for surgery on an acute proximal dissection involving the aortic arch remains debatable. For example, should patients with dissection beyond the ascending aorta (Stanford type B) undergo surgery if the arch is involved? Most agree that surgery is not initially required for those patients with type $\mathrm{B}$ dissection beyond the left subclavian artery. Moreover, in those patients with type A dissection, involving the ascending aorta and arch (excluding DeBakey type II), should a more aggressive arch reconstruction be performed? Certainly, earlier literature from Stanford and Baylor College of Medicine concurred that arch replacement should not be performed unless there is a clear indication, such as arch rupture or a large

From the Department of Thoracic and Cardiovascular Surgery, Cleveland Clinic, Cleveland, Ohio.

Disclosures: Author has nothing to disclose with regard to commercial support.

Received for publication Sept 29, 2014; accepted for publication Oct 1, 2014.

Address for reprints: Lars G. Svensson, MD, PhD, Department of Thoracic and Cardiovascular Surgery, Cleveland Clinic, 9500 Euclid Ave, Cleveland, OH 44195 (E-mail: svenssl@ccf.org).

J Thorac Cardiovasc Surg 2014;148:2138-9

0022-5223/ $\$ 36.00$

Copyright $₫ 2014$ Published by Elsevier Inc. on behalf of The American Association for Thoracic Surgery

http://dx.doi.org/10.1016/j.jtcvs.2014.10.003 arch aneurysm. Also, even tears in the arch can be oversewn and the ascending and hemiarch replaced with minimization of the extent of surgery and circulatory arrest time. More recently, the "frozen elephant trunk" approach has been adopted, with various combinations of retrograde or antegrade delivery of stent grafts in the descending thoracic aorta. The general consensus is that this will reduce the risk of subsequent reoperations, and indeed there is some evidence for this. Nonetheless, this approach likely carries the risk of longer circulatory arrest time and more strokes, with a greater risk of death. There would be an expected number of patients who have gut ischemia also. In the German Registry of Acute Aortic Dissection Type A (GERAADA), among 658 patients with ascending aortic tears, 518 underwent ascending hemiarch and 140 total arch replacement with or without elephant trunk or frozen elephant trunk. As expected, circulatory arrest time was 24.3 minutes versus 44.8 minutes $(P<.001)$, mortality was $18.7 \%$ versus $25.7 \%(P=.07)$, and neurologic deficit was $13.6 \%$ versus $12.5 \%(P=.78)$; however, in those patients without preoperative neurologic deficits, there was a significant difference in mortality $(14.1 \%$ vs $24 \% ; P=.02)$. The multivariable logistic predictors of death were age, resuscitation, brain perfusion time, and circulatory arrest time, emphasizing the increased risk associated with the more complex repairs that may reduce later risk of reoperation.

In this issue of the Journal, $\mathrm{Wu}$ and colleagues report excellent results (mortality, 3.2\%; stroke, 1.6\%) with a 